Article

\title{
Poly(lactide-co-glycolide)/Hydroxyapatite Porous Scaffold with Microchannels for Bone Regeneration
}

\author{
Ning Zhang ${ }^{1,2}$, Yang Wang ${ }^{1}$, Wenpeng $\mathrm{Xu}^{1}$, Yong $\mathrm{Hu}{ }^{1, *}$ and Jianxun Ding ${ }^{2, *}$ \\ 1 Department of Foot and Ankle Surgery, The Second Hospital of Shandong University, Jinan 250033, China; \\ zhangningno1@126.com (N.Z.); handfootsurgery@126.com (Y.W.); chinaowenxu@126.com (W.X.) \\ 2 Changchun Institute of Applied Chemistry, Chinese Academy of Sciences, Changchun 130021, China \\ * Correspondence: anklesurgeonhu@gmail.com (Y.H.); jxding@ciac.ac.cn (J.D.); \\ Tel.: +86-531-8587-5163 (Y.H.); +86-431-8526-2116 (J.D.)
}

Academic Editor: Carsten Werner

Received: 7 April 2016; Accepted: 30 May 2016; Published: 7 June 2016

\begin{abstract}
Mass transfer restrictions of scaffolds are currently hindering the development of three-dimensional (3D), clinically viable, and tissue-engineered constructs. For this situation, a 3D poly(lactide-co-glycolide)/hydroxyapatite porous scaffold, which was very favorable for the transfer of nutrients to and waste products from the cells in the pores, was developed in this study. The 3D scaffold had an innovative structure, including macropores with diameters of 300-450 $\mu \mathrm{m}$ for cell ingrowth and microchannels with diameters of $2-4 \mu \mathrm{m}$ for nutrition and waste exchange. The mechanical strength in wet state was strong enough to offer structural support. The typical structure was more beneficial for the attachment, proliferation, and differentiation of rabbit bone marrow mesenchymal stem cells (rBMSCs). The alkaline phosphatase (ALP) activity and calcium (Ca) deposition were evaluated on the differentiation of rBMSCs, and the results indicated that the microchannel structure was very favorable for differentiating rBMSCs into maturing osteoblasts. For repairing rabbit radius defects in vivo, there was rapid healing in the defects treated with the $3 \mathrm{D}$ porous scaffold with microchannels, where the bridging by a large bony callus was observed at 12 weeks post-surgery. Based on the results, the 3D porous scaffold with microchannels was a promising candidate for bone defect repair.
\end{abstract}

Keywords: poly(lactide-co-glycolide); hydroxyapatite; porous scaffold; microchannel; cell ingrowth; mass exchange; bone tissue engineering

\section{Introduction}

Recently, orthopedic reconstruction procedures stemming from trauma, tumor, deformity, degeneration, and an aging population have dramatically increased, triggering a high demand on the improvement of bone implant technology [1,2]. Modern clinical practice in orthopedics has demonstrated that the autograft exhibits a superior therapeutic effect in bone fusion. However, donor site morbidity and limited supply are major concerns. Allografts and xenografts may raise other concerns in pathogen transmission and immunorejection, respectively [3,4]. Therefore, the development of synthetic materials for musculoskeletal tissue engineering is paramount in order to satisfy the booming demand of increased orthopedic implantations.

In the field of musculoskeletal tissue engineering, a plethora of biodegradable tissue substitutes have been created and tested, and some early products were marketed. Due to the complex structure required for three-dimensional (3D) thicker tissues [5], science is some way off from generating a definitive clinical product for bone tissue regeneration. This is largely due to the non-homogeneous growth of cells on the traditional "porous block" scaffolds, which prevent the formation of a functional construct from surface to core. It is due to the mass transfer limitations of the porous structures, into 
which cells are expected to invade and populate. The movement of nutrients to and waste products from the cells in the pores depends on molecular diffusion; nutrients are used up before reaching the inner core of the construct, and waste products build up. Cells that do migrate into the core become necrotic, and so the cell population is commonly found to be concentrated at the periphery of the scaffold [6].

Dunn et al. introduced an in situ solidification system composed of a poly(lactide-co-glycolide) (PLGA) implant in the 1990s [7]. This implant was dissolved in water-miscible solvents, such as $\mathrm{N}$-methyl-2-pyrrolidone (NMP) and dimethyl sulfoxide (DMSO). The solid implants were formed in situ due to phase inversion triggered by solvent/non-solvent exchange. After contact with the aqueous medium, the high water miscibility of the solvents resulted in a fast phase inversion of the polymer solutions, and thus solidification of the system took place in seconds to minutes. Also, because of the phase inversion, a microchannel structure was created [8-10]. For example, Ellis et al. produced PLGA flat sheet membranes with a finger-like structure using NMP as a solvent and water as a non-solvent $[6,11]$. Porous structures are expected to form in the high mutual affinity of the NMP-water medium. Oh et al. fabricated the hydrophilic porous PLGA tubes using a modified immersion phase-inversion method and showed that the tubes were highly effective for the permeation of bovine serum albumin (BSA) [12]. These studies inspired us to devise a 3D porous scaffold with microchannels for bone repair via the phase inversion method to improve mass transport.

In this study, we fabricated an innovative 3D porous scaffold by phase inversion/particulate leaching method (PI), which possessed both macropores and microchannels, providing space for cell invasion and mass transfer, respectively. In our lab, the 3D porous scaffolds by melt-molding/particulate leaching method (MM) were studied for many years [13]. In order to explore the advantage of PI scaffold $\left(\mathrm{S}_{\mathrm{PI}}\right)$, the scaffold fabricated by $\mathrm{MM}\left(\mathrm{S}_{\mathrm{MM}}\right)$ was applied to compare it in terms of structure, porosity, mechanical property, cell attachment, cell proliferation, osteogenic differentiation, and the capability of bone repair in vivo.

\section{Materials and Methods}

\subsection{Preparation of PLGA/HA Homogeneous Hybrid Composite}

The homogeneous hybrid composite composed of PLGA (Viscosity-average molecular weight $\left(M_{\eta}\right)=147,000 \mathrm{Da}, \mathrm{LA}: \mathrm{GA}=75: 25$ (mol/mol), Changchun SinoBiomaterials Co., Ltd., Changchun, China) and hydroxyapatite (HA) (Nanjing Emperor Nano Material Co., Ltd., Nanjing, China) (HA:PLGA $=1: 9(W / W)$ ) was prepared by the solvent-mixing method. Briefly, HA powder was uniformly suspended in 20-fold (by wt \%) chloroform by magnetic stirring and ultrasonic treatment. The suspension was added into a 5\% PLGA/chloroform solution to achieve the $10 \mathrm{wt} \% \mathrm{HA}$ in the hybrid composite. The mixture was precipitated in an excess of ethanol, and the composite was dried in air for $48 \mathrm{~h}$ and vacuumed for $72 \mathrm{~h}$ to remove the residual solvent.

\subsection{PLGA/HA Scaffold Fabrication via PI Method}

A PLGA/HA scaffold was fabricated via combining phase inversion and particulate leaching $\left(\mathrm{S}_{\mathrm{PI}}\right)$. Firstly, two grams of PLGA/HA hybrid composite were added into $10.0 \mathrm{~mL}$ of NMP (Aladdin, Shanghai, China). After magnetic stirring under $70^{\circ} \mathrm{C}$ for $6 \mathrm{~h}$, the homogeneous PLGA/HA/NMP mixture was obtained. Next, the sieved sodium chloride particulates of 300-450 $\mu \mathrm{m}$ in diameter were added into the PLGA/HA/NMP mixture. The weight ratio of the salt particulates to PLGA/HA was 6:1. The mixture was cast in a homemade glass cylinder with a removable bottom. To remove NMP and salt particulates, the bottom was removed, and the mixture was immersed in distilled water for three days with the water exchanged every $12 \mathrm{~h}$. Subsequently, any water remaining in the scaffold was exchanged by ethanol. Finally, the PLGA scaffold was obtained after three days of lyophilization and sterilized with ethylene oxide for $6 \mathrm{~h}$. In addition, A PLGA/HA film was also fabricated. In detail, a drop of the PLGA/HA/NMP mixture were laid on the $\Phi 8 \mathrm{~mm}$ round siliconized slide and then 
smeared on the surface homogeneously. Subsequently, the coated slides were immersed in $500.0 \mathrm{~mL}$ of distilled water for $10 \mathrm{~min}$ to remove NMP. The obtained PLGA/HA film-coated slide by phase inversion $\left(\mathrm{F}_{\mathrm{PI}}\right)$ would be used for cell culture in the next.

\subsection{PLGA/HA Scaffold Fabrication via MM Method}

A PLGA/HA scaffold was fabricated by a melt-molding/particulate leaching method $\left(\mathrm{S}_{\mathrm{MM}}\right)$. Briefly, the sieved sodium chloride particulates of 300-450 $\mu \mathrm{m}$ in diameter were added into the melted HA/PLGA hybrid composite in an internal mixer at $150{ }^{\circ} \mathrm{C}$ and $60 \mathrm{rpm}$. The weight ratio of salt particulates to the composite was 6:1. The obtained mixture was then molded into 3-mm-thick sheets under $10 \mathrm{MPa}$ pressure at $150^{\circ} \mathrm{C}$ for $5 \mathrm{~min}$, and then cooled to room temperature. The salt particles were removed from the molds by leaching in distilled water for two weeks, and the water was changed every $12 \mathrm{~h}$. Finally, the porous scaffolds were obtained after dried in air for $48 \mathrm{~h}$ and vacuumed for $72 \mathrm{~h}$ to remove the residual solvent, and sterilized with ethylene oxide for $6 \mathrm{~h}$. In addition, a PLGA/HA film was also fabricated by melt-molding method ( $\left.\mathrm{F}_{\mathrm{MM}}\right)$ as process of $\mathrm{F}_{\mathrm{PI}}$ fabrication.

\subsection{Characterizations of Scaffolds}

The porosities of scaffolds were determined using the ethanol replacement method. The microstructures of the scaffolds were examined by scanning electron microscopy (SEM; Philips XL30, Philips, Amsterdam, The Netherlands). The scaffolds were fractured after snap-freezing, sputter-coated with gold, and observed at an accelerating voltage of $15 \mathrm{kV}$. For characterizing the distribution and exposure degrees of HA in PLGA matrix, it was analyzed with energy dispersive X-ray spectrometry (EDX) (XL-30W/TMP, Philips, Amsterdam, The Netherlands). Rectangular bars of $30 \mathrm{~mm} \times 5 \mathrm{~mm} \times 5 \mathrm{~mm}$ in dry and wet state were chosen for mechanical strength tests measured by a universal testing machine (Instron 1121, Norwood, MA, USA). The compressive strength was measured at a crosshead speed of $2 \mathrm{~mm} / \mathrm{min}$. The stress histogram was obtained to determine mechanical properties. Three replicates were tested for the wet and dry conditions $(n=3)$.

\subsection{Cell Culture}

\subsubsection{Isolation of Rabbit Bone Marrow Mesenchymal Stem Cells (rBMSCs)}

Three-month-old New Zealand white rabbits were selected for rBMSC isolation according to an established protocol. The animals were provided by Jilin University, Changchun, China, and treated according to the NIH Guide for the Care and Use of Laboratory Animals (NIH Publication No. 85-23, revised 1996). Bone marrow aspirates $(5 \mathrm{~mL})$ were obtained from rabbit tibia and subsequently cultured. Briefly, the isolated cell pellets were resuspended in $5.0 \mathrm{~mL}$ of culture medium (DMEM; Dulbecco's Modified Eagle Medium (Gibco, Carlsbad, CA, USA) supplemented with 10\% $(\mathrm{V} / \mathrm{V})$ fetal calf serum (Gibco, Carlsbad, CA, USA) and $100 \mathrm{IU} / \mathrm{mL}$ penicillin-streptomycin (Sigma, Shanghai, China)). The cells were seeded in culture dishes (Corning Costar Co., Cambridge, MA, USA) and cultured in a $37{ }^{\circ} \mathrm{C}$ and $5 \%$ carbon dioxide $\left(\mathrm{CO}_{2}\right)$ incubator. Non-adherent cells were removed when the medium was changed after $24 \mathrm{~h}$. After that, the medium was replaced every three days until the cells reached $80 \%$ confluence. Then, the cells were washed twice with phosphate-buffered saline (PBS), detached by treatment with $0.25 \%$ trypsin-ethylenediamine tetraacetic acid (EDTA, Sigma, Shanghai, China), and subcultured under the same condition until the third passage.

\subsubsection{Cell Adhesion}

For adhesion studies, $\mathrm{rBMSC}$ s were seeded onto $\mathrm{F}_{\mathrm{PI}}, \mathrm{F}_{\mathrm{MM}}$, and glass at an initial seeding density of $1 \times 10^{4}$ cells $/ \mathrm{cm}^{2}$ and incubated for 4,10 , and $24 \mathrm{~h}$. The cells were washed three times with phosphate-buffered saline (PBS), fixed with $4 \%(\mathrm{~W} / \mathrm{V})$ paraformaldehyde (PFA) at room temperature for $10 \mathrm{~min}$, dyed with $2 \%$ fluorescein isothiocyanate (FITC) DMSO/ $\mathrm{H}_{2} \mathrm{O}$ solution for $10 \mathrm{~min}$, and then washed with PBS three times. Cell attachment was observed qualitatively under a reverse microscope 
(TE2000U, Nikon, Tokyo, Japan). The fluorescence pictures were taken by Digital Camera DXM1200F (Nikon, Tokyo, Japan) and analyzed with “NIH ImageJ" software (>15 per sample).

\subsubsection{Cell Proliferation}

To investigate the cell proliferation on $\mathrm{F}_{\mathrm{PI}}, \mathrm{F}_{\mathrm{MM}}, \mathrm{S}_{\mathrm{PI}}$, and $\mathrm{S}_{\mathrm{MM}}$, rBMSCs were cultured on the two-dimensional (2D) films ( $\mathrm{F}_{\mathrm{PI}}$ and $\mathrm{F}_{\mathrm{MM}}$ ) at a density of $1 \times 10^{4}$ cells $/ \mathrm{cm}^{2}$ and 3D scaffolds $\left(\mathrm{S}_{\mathrm{PI}}\right.$ and $\mathrm{S}_{\mathrm{MM}}$ ) at a density of $2 \times 10^{4} \mathrm{cells} / \mathrm{cm}^{2}$. After the indicated incubation times, the medium was replaced by Cell Counting Kit-8 (CCK-8, Dojindo, Japan). After $3 \mathrm{~h}$ of incubation, the absorbance values at $450 \mathrm{~nm}$ were measured on multifunction microplate scanner (Tecan Infinite M200, Männedorf, Zürich, Switzerland).

\subsubsection{Cell Differentiation}

Alkaline phosphatase (ALP) activity was determined after culturing the cells in DMEM/F12, FBS $(10 \%, V / V)$ for five and 10 days. Briefly, the medium of each well was carefully removed. Then, the cells were washed with PBS three times, lysed in radioimmunoprecipitation assay (RIPA) buffer, frozen at $-80{ }^{\circ} \mathrm{C}$ for $30 \mathrm{~min}$, and thawed at $37^{\circ} \mathrm{C}$. Then, $p$-nitrophenol phosphate substrate $(p N P P)$ solution (Aladdin, Shanghai, China) was added, and the samples were incubated in the dark for $30 \mathrm{~min}$ at $37^{\circ} \mathrm{C}$. The reaction was terminated with $3.0 \mathrm{M}$ sodium hydroxide $(\mathrm{NaOH})$, and the ALP activity was read on a multifunction microplate scanner at $405 \mathrm{~nm}$.

Calcium (Ca) deposition was determined by alizarin red S (ARS) staining of the rBMSCs after culture in DMEM/F12, FBS $(10 \%, V / V)$ for 14 and 21 days. After three 5 min rinses in water, the scaffolds were incubated in ARS stain solution (0.1\% ARS in Tris-HCl buffer, $\mathrm{pH}$ 8.0, Sigma-Aldrich, St. Louis, MI, USA) for $30 \mathrm{~min}$ at $37^{\circ} \mathrm{C}$. The scaffolds were then washed in distilled water three times for $5 \mathrm{~min}$ each. The stained samples were treated with $10 \%(\mathrm{~W} / \mathrm{V})$ cetylpyridinium chloride in $10.0 \mathrm{mM}$ sodium phosphate for $15 \mathrm{~min}$ at room temperature. The absorbance of ARS at $540 \mathrm{~nm}$ was recorded on a multifunction microplate scanner.

\subsection{In Vivo Animal Study}

\subsubsection{Implantation for Radius Defect Repair}

Bilateral critically sized defects of nine rabbits were created with saw and drill in the radius of each rabbit forelimb by removing $2.0 \mathrm{~cm}$ of midshaft diaphyseal bone. A total of 18 radius defects were randomly divided into three groups with six defects treated with $S_{P I}$ and $S_{M M}$, respectively, and six defects in blank group as control.

The porous scaffold bars $\left(0.3 \mathrm{~cm}\right.$ in width, $2.0 \mathrm{~cm}$ in length) of $\mathrm{S}_{\mathrm{PI}}$ and $\mathrm{S}_{\mathrm{MM}}$ were placed into the defects of different rabbits, separately. The wounds were closed with silk threads in layers. After surgery, the rabbits were returned to their cages and allowed to move freely. All rabbits were injected daily with penicillin intramuscularly in a dose of 200,000 units for each one for three days. All the wounds healed gradually and the rabbits were active with no post-surgery complications.

\subsubsection{X-ray Examination}

In vivo osteogenesis at the rabbit radius defects repaired with $S_{\mathrm{PI}}$ and $\mathrm{S}_{\mathrm{MM}}$ were examined with Digital Radiograph (DR, Philips Digital diagnost, Philips, Amsterdam, The Netherlands) at baseline and 12 weeks post-surgery. The rabbits were exposed to $\mathrm{X}$-ray in prone position during anesthetization. Afterwards, X-ray films were exported as TIF images. The newly formed bone was identified for quantifying its size and calculating its area fraction within the proportional area of its original bone defect region using a free and open ImageJ software developed by National Institutes of Health. All X-rays films were scored by the Lane-Sandhu scoring system [14]. 


\subsection{Statistical Analyses}

The data were presented as mean \pm standard deviation (SD). The independent and replicated experiments were used to analyze the statistical variability of the data analyzed using Student's $t$-test. $p<0.05$ was considered to be statistically significant, and $p<0.01$ and $p<0.001$ were considered to be highly significant.

\section{Results and Discussion}

\subsection{Scaffold Characterizations}

The microstructures of $\mathrm{S}_{\mathrm{PI}}$ and $\mathrm{S}_{\mathrm{MM}}$ were analyzed with SEM (Figure 1). Figure 1 shows that both fabricated scaffolds have the irregular macropores, and most of them are interconnected, which was suited for cell infiltration. The opening porosities of $S_{P I}$ and $S_{M M}$ are $87.27 \% \pm 6.84 \%$ and $85.72 \% \pm 9.45 \%$, and there are no significant differences statistically $(p>0.05)$. However, the architecture of the pore surface and wall were different between them. As shown in Figure 2, the wall of $S_{P I}$ had a honeycomb-like structure composed of microvoids with diameters of $2-4 \mu \mathrm{m}$, and the surface displayed microscale channels to which the internal macropores and microvoids in the skeleton were connected. The observed architecture was very favorable for the movement of proteins [12]. In contrast, the $S_{M M}$ showed a smooth pore surface and solid pore wall, which impeded the material exchange.

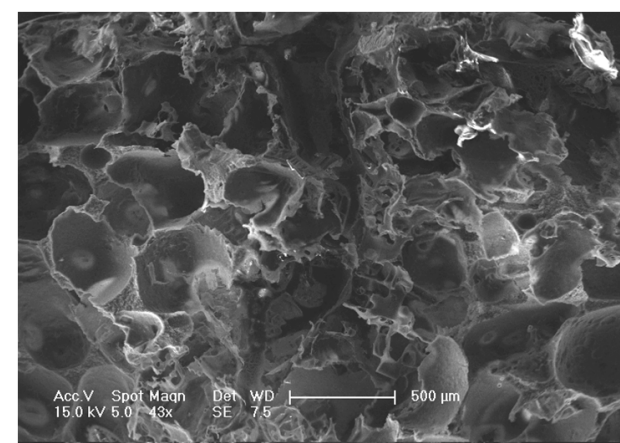

(A)

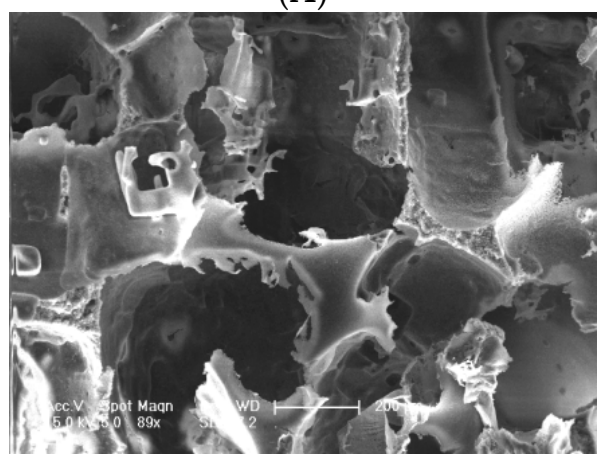

(C)

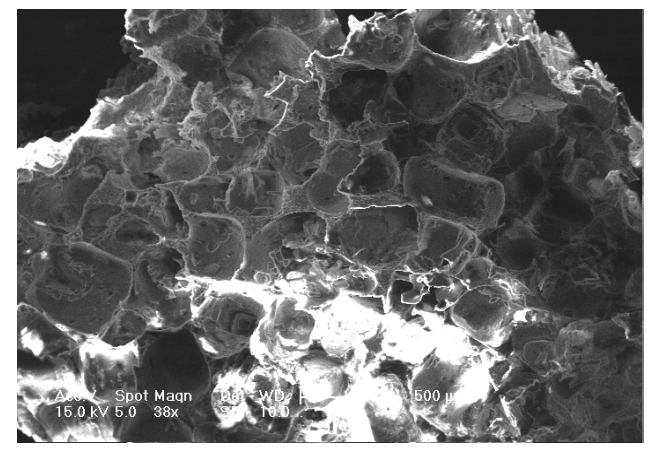

(B)

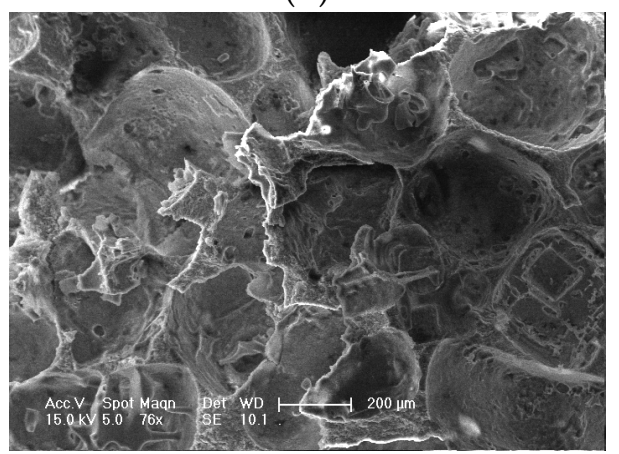

(D)

Figure 1. SEM images of $\mathrm{S}_{\mathrm{PI}}(\mathbf{A}, \mathbf{C})$ and $\mathrm{S}_{\mathrm{MM}}$ microstructures $(\mathbf{B}, \mathbf{D})$. The bar lengths are $500 \mu \mathrm{m}(\mathbf{A}, \mathbf{B})$ and $200 \mu \mathrm{m}(\mathbf{C}, \mathbf{D})$, respectively.

The formation of microscale channels and microvoids in $\mathrm{S}_{\mathrm{PI}}$ is based on the phase separation and gelation behavior of a PLGA/HA/NMP solution. When PLGA/HA/NMP solution comes into contact with a non-solvent, the influx of the non-solvent into the surface results in a process of phase separation, whereby the solution separates into a polymer-rich and polymer-lean phase [15]. Eventually, the concentration of the polymer in the polymer-rich phase becomes high enough for 
gelation to occur. This results in "freezing" of the two-phase structure, leading to microscale channels and microvoids.

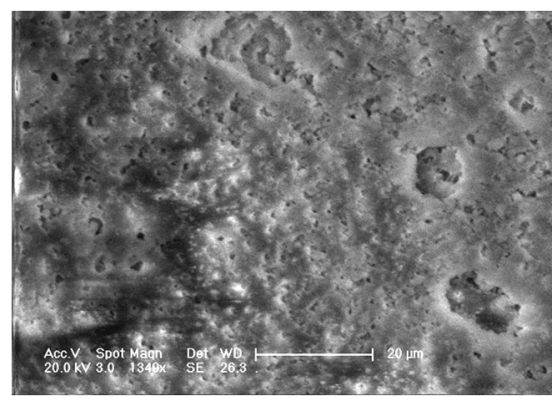

(A)

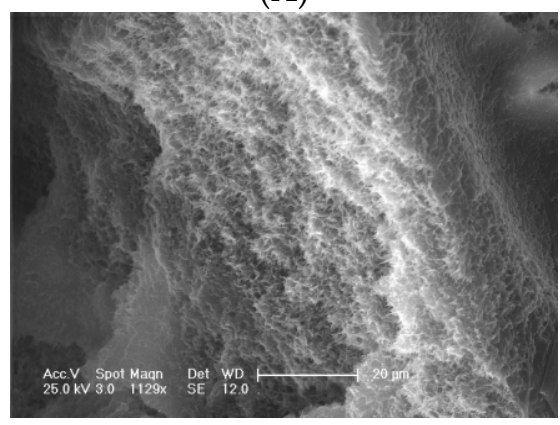

(C)

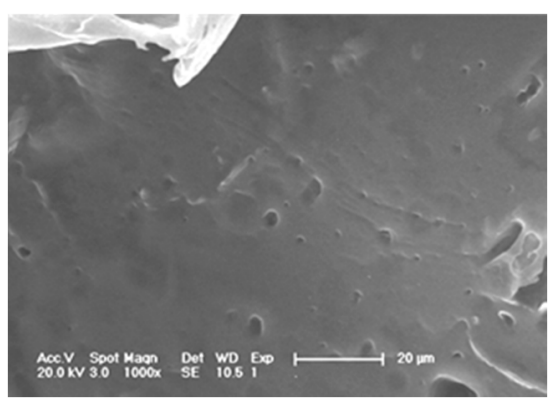

(B)

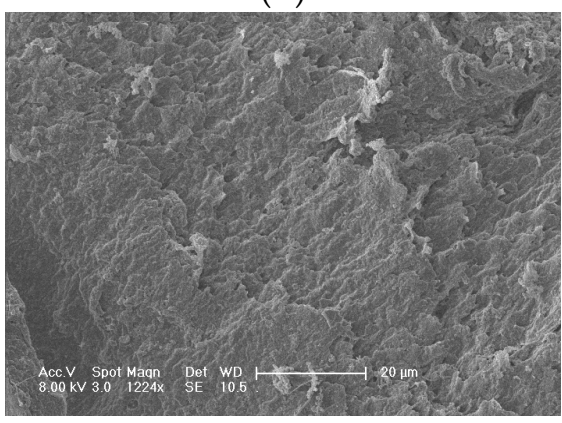

(D)

Figure 2. SEM images of pore surface and wall of $S_{P I}(A, C)$ and $S_{M M}(\mathbf{B}, \mathbf{D})$. The bar length is $20 \mu \mathrm{m}$.

EDX element analysis was used to assess the content of calcium (Ca), phosphorous (P), and oxygen $(\mathrm{O})$ elements on the surface of the porous scaffolds. The EDX spectra of $\mathrm{S}_{P I}$ and $\mathrm{S}_{\mathrm{MM}}$ are illustrated in Figure 3. The presence of HA in $\mathrm{S}_{\mathrm{PI}}$ and $\mathrm{S}_{\mathrm{MM}}$ was confirmed by the appearance of the characteristic peaks of $\mathrm{Ca}, \mathrm{P}$, and $\mathrm{O}$, which are the main components of $\mathrm{HA}$, while the $\mathrm{Ca}$ and $\mathrm{P}$ exposures in $\mathrm{S}_{\mathrm{PI}}$ and $\mathrm{S}_{\mathrm{MM}}$ were different. The weight percents (wt \%) of $\mathrm{Ca}$ and $\mathrm{P}$ exposed on the surface of $S_{P I}$ were 8.21 and $5.42 \mathrm{wt} \%$, which were higher than those on the $S_{M M}$ scaffold, i.e., 5.92 and $3.4 \mathrm{wt} \%$, respectively. This could be due to the microchannels in the surface, which increase the HA exposure.

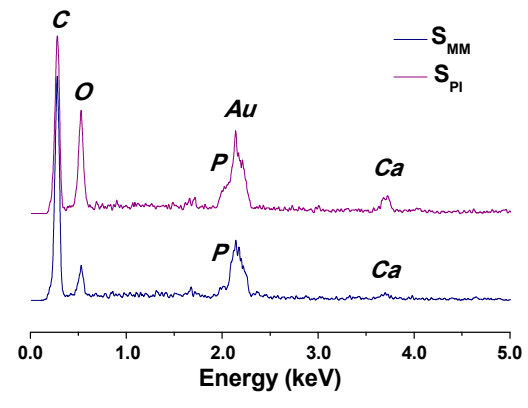

(A)

\begin{tabular}{|c|c|c|}
\hline & $\mathrm{S}_{\mathrm{MM}}$ & $\mathrm{S}_{\mathrm{PI}}$ \\
\hline Element & Wt \% & Wt \% \\
\hline CK & 51.17 & 34.39 \\
\hline $\mathrm{OK}$ & 4.42 & 3.53 \\
\hline $\mathbf{P K}$ & 3.4 & 5.42 \\
\hline AuM & 35.09 & 48.45 \\
\hline CaK & 5.92 & 8.21 \\
\hline Total & 100 & 100 \\
\hline
\end{tabular}

(B)

Figure 3. EDX spectra (A) and element contents (B) of $\mathrm{S}_{\mathrm{PI}}$ and $\mathrm{S}_{\mathrm{MM}}$.

The porous scaffolds are designed to provide mechanical support until the regenerative tissue or organ is structurally stabilized [16]. Therefore, the appropriate mechanical properties are crucial for such porous scaffolds. For instance, the fibrous scaffolds done by electrospinning also possess 
the interconnected structure for mass transferring, though they were rarely applied for bone defect repair in the weight-bearing area because of their poor mechanical property [17,18]. The scaffold should maintain its structural stability and integrity in an in vivo biomechanical environment and provide appropriate microstress stimulations for the implanted cells [19]. The mechanical property was often measured in the dry state. The compressive strength and elastic modulus were $1.31 \pm 0.14$ and $5.63 \pm 0.51 \mathrm{MPa}$ for $\mathrm{S}_{\mathrm{PI}}$ and $1.80 \pm 0.19$ and $7.08 \pm 0.97 \mathrm{MPa}$ for $\mathrm{S}_{\mathrm{MM}}$ (Figure 4), but they were different from that under physiological conditions, i.e., in tissue fluid at $37^{\circ} \mathrm{C}$, because of the different media. As depicted in Figure 4, in wet state, the compressive strength and elastic modulus of $\mathrm{S}_{\mathrm{PI}}$ reached $1.01 \pm 0.11$ and $2.46 \pm 0.90 \mathrm{MPa}$, respectively, which is strong enough to offer structural support, and they are similar to those of $S_{\mathrm{MM}}(1.17 \pm 0.13$ and $2.37 \pm 0.50 \mathrm{MPa})$ (Figure 4$)$.

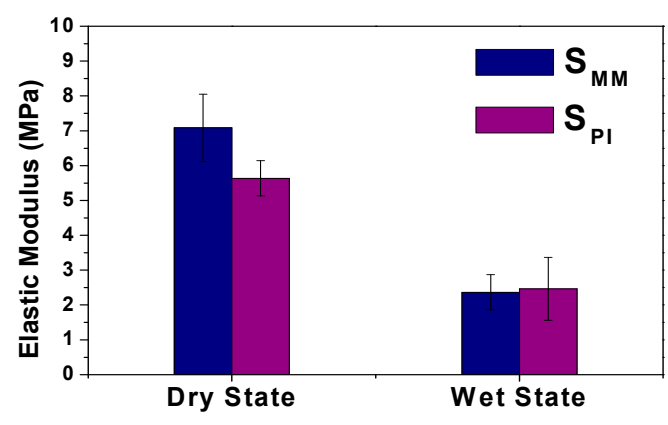

(A)

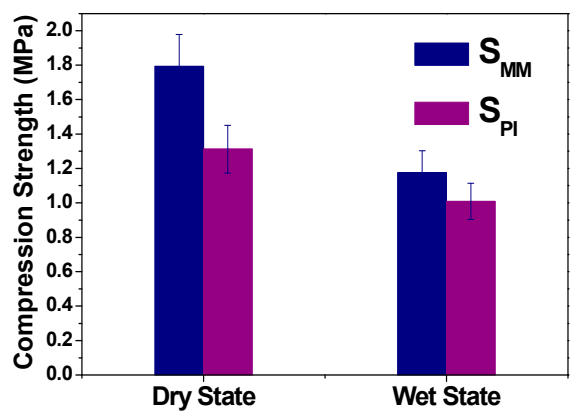

(B)

Figure 4. Elastic modulus (A) and compression strength (B) of $S_{M M}$ and $S_{P I}$ in dry and wet states.

\subsection{Cell Adhesion, Proliferation, and Differentiation}

The behaviors of rBMSCs on $\mathrm{F}_{\mathrm{PI}}$ and $\mathrm{F}_{\mathrm{MM}}$ were investigated compared to a control substrate of glass. The cells were seeded and cultured for 4,10 , and $24 \mathrm{~h}$ on different substrates to assess cell adhesion (Figure 5). With the increase of culture time, the average area of cells on glass was highest, and the cells showed the best spread due to their good hydrophilicity. For $\mathrm{F}_{\mathrm{PI}}$ and $\mathrm{F}_{\mathrm{MM}}$, the cells also showed different behaviors. Cells on $\mathrm{F}_{\mathrm{PI}}$ showed an elongated and spindle-like morphology after culture for $4 \mathrm{~h}$ and became a cuboidal morphology after $10 \mathrm{~h}$. However, the cells on $\mathrm{F}_{\mathrm{MM}}$ did not extend until culturing for $10 \mathrm{~h}$. The average area of cells on $\mathrm{F}_{\mathrm{PI}}$ was also much higher than that on $\mathrm{F}_{\mathrm{MM}}$. It was believed that the roughness and topography of the pore surface increased direct cell-material binding, thus facilitating an increase in early cell adhesion [20,21]. After $24 \mathrm{~h}$, the adhesive rates were similar between $\mathrm{F}_{\mathrm{PI}}$ and $\mathrm{F}_{\mathrm{MM}}$. The results suggested that the cell adhesion rate was related to the surface characteristics of materials, including stiffness, roughness, and hydrophilicity.
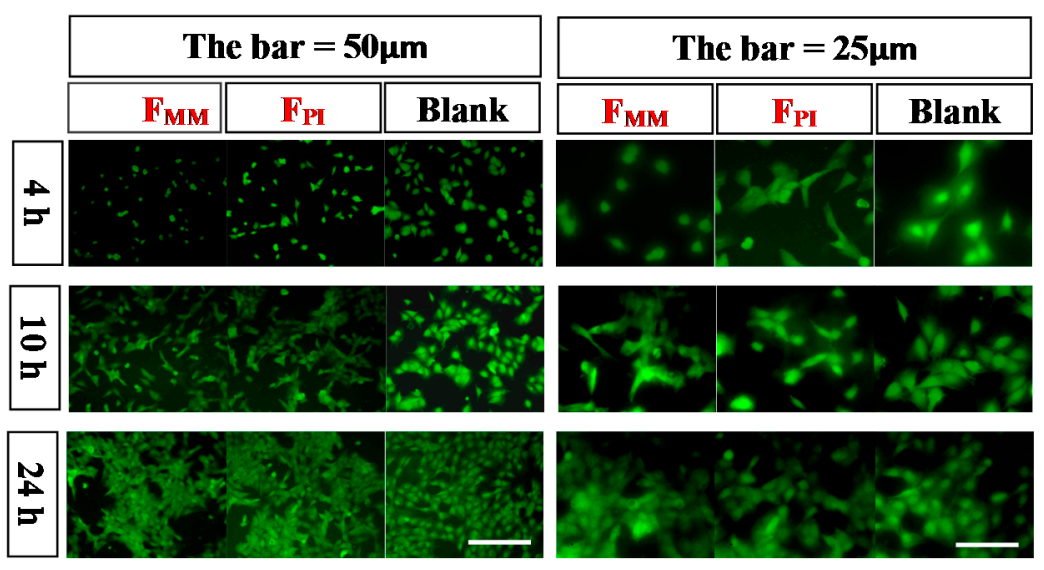

Figure 5. Cell adhesion on different substrates for 4, 10, and $24 \mathrm{~h}$. 
The cell proliferation on the films $\left(\mathrm{F}_{\mathrm{PI}}\right.$ and $\left.\mathrm{F}_{\mathrm{MM}}\right)$ and scaffolds $\left(\mathrm{S}_{\mathrm{PI}}\right.$ and $\left.\mathrm{S}_{\mathrm{MM}}\right)$ was monitored quantitatively via CCK-8 assay to determine the metabolic activity of the total population of cells (Figure 6). After rBMSCs become attached to the surface, they enter a rapid proliferative growth phase in order to establish critical cell-cell interactions essential for the subsequent post-confluent differentiation growth phase. After culturing for three and five days on $\mathrm{F}_{\mathrm{PI}}$ and $\mathrm{F}_{\mathrm{MM}}$ films, rBMSCs showed significantly sustained growth. However, after 10 days of the cell seeding, the metabolic activity of the cells was not higher than that at five days in culture. It was deduced that the two-dimensional (2D) film cannot provide sufficient space for cell growth. In addition, there is no difference between $\mathrm{F}_{\mathrm{PI}}$ and $\mathrm{F}_{\mathrm{MM}}$ on cell proliferation during 10 days in culture. It means that the roughness of $\mathrm{F}_{\mathrm{PI}}$ did not accelerate the growth of rBMSCs, which is not in accordance with the previous studies [20]. The cell proliferation on 3D scaffolds was different with that on $2 \mathrm{D}$ films. For $\mathrm{S}_{\mathrm{MM}}$, the population of cells was significantly increased from Day 3 to 5 and increased slightly from Day 5 to 10 , while the cells cultured on $S_{\text {PI }}$ grew continuously from Day 3 to 10. Moreover, the metabolic activity of cells was higher than $\mathrm{F}_{\mathrm{MM}}$ on Day 10. In brief, $\mathrm{F}_{\mathrm{PI}}$ did not display better ability for cell growth compared to $\mathrm{F}_{\mathrm{MM}}$, when rBMSCs were cultured on 2D films for 10 days. However, 3D $\mathrm{S}_{\mathrm{PI}}$ improved the cell proliferation with respect to $S_{M M}$. These results suggested that $3 D S_{M M}$ lacks nutrition in the core of the scaffold after five days. Conversely, the special microstructure of $S_{P I}$ was very favorable for the cell population in the core because the microchannels on the surface sped up the exchange of nutrients to and waste products from the cells in the inner scaffold.

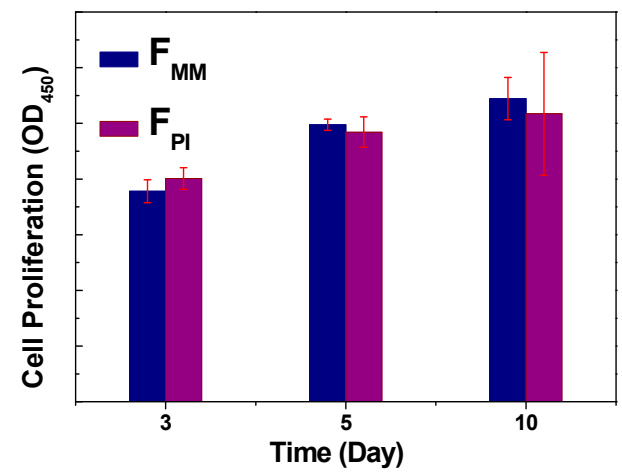

(A)

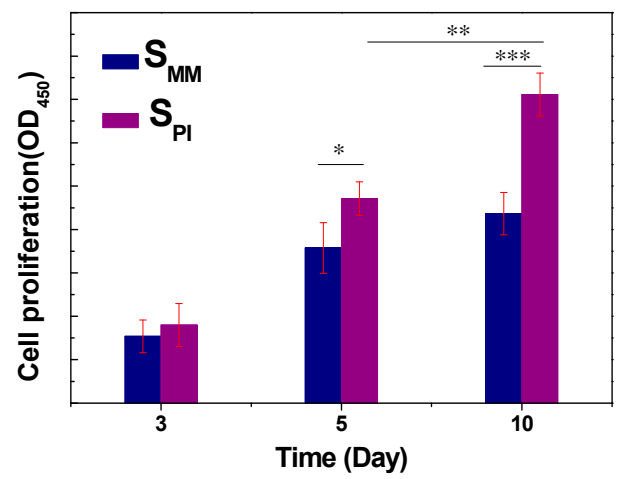

(B)

Figure 6. Cell proliferation on different films (A) and scaffolds (B) for three, five, and 10 days. Data are presented as mean $\pm \mathrm{SD}\left(n=6,{ }^{*} p<0.05 ;{ }^{* *} p<0.01 ;{ }^{* * *} p<0.001\right)$.

The cell-biomaterial interactions have been demonstrated to exert a considerable influence on the differentiation and function of BMSCs [22]. To investigate the osteogenic differentiation of rBMSCs on $S_{P I}$ and $S_{M M}$, ALP activity and Ca deposition were measured. ALP is a membrane enzyme commonly recognized as a marker of osteoblastic differentiation. Figure 7A shows the ALP activities of the rBMSCs cultured on $S_{P I}$ and $S_{M M}$ after five and 10 days. The significantly higher ALP activity was detected in cells cultured on $\mathrm{S}_{\mathrm{PI}}$ compared to $\mathrm{S}_{\mathrm{MM}}$ on Day $10(p<0.05)$. Ca deposition was measured by ARS. As shown in Figure 7B, the quantification of ARS indicated that the deposition of Ca minerals in the $S_{P I}$ was significantly higher than that in $S_{M M}$ after culture for 14 and 21 days $(p<0.05)$. All results suggested that the cell differentiation toward osteogenesis was better on $\mathrm{S}_{\mathrm{PI}}$ than $\mathrm{S}_{\mathrm{MM}}$. It is well accepted that the surface structures, such as topographies, roughness, and nano-architectures, influence the cell and tissue behaviors of biomaterials [23,24]. So it is deduced that the stiffness and microchannels regulated cell proliferation. 


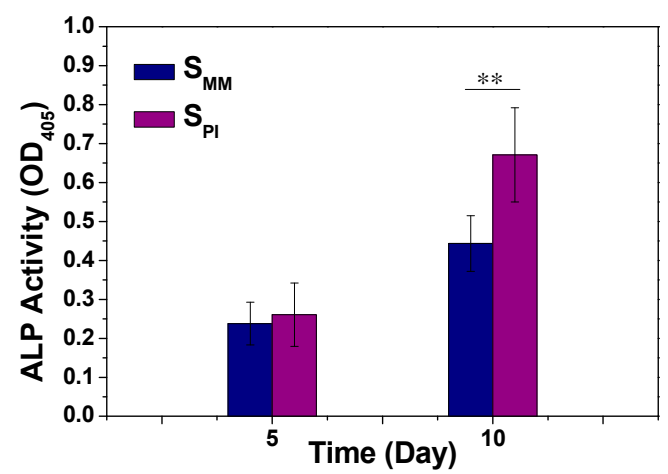

(A)

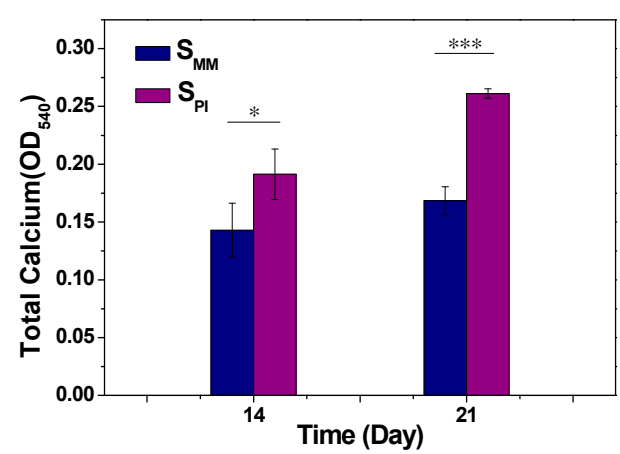

(B)

Figure 7. ALP activity (A) and Ca deposition (B) of $\mathrm{S}_{\mathrm{MM}}$ and $\mathrm{S}_{\mathrm{PI}}$. Data are presented as mean $\pm \mathrm{SD}$ $\left(n=6,{ }^{*} p<0.05 ;{ }^{* *} p<0.01 ; * * p<0.001\right)$.

\subsection{Radius Bone Defect Repair}

At 12 weeks post-surgery, the defects without scaffold implantation are only filled with scattered bony structure (Figure 8). Bone defects implanted with $\mathrm{S}_{\mathrm{MM}}$ revealed that the new bone tissue was concentrated at the periphery of the scaffold (Figure 8), which indicated that the osteocyte did not invade into the inner core of $S_{M M}$ in the beginning, or the cells in the inner core were necrotic because of nutrition deficiency. In contrast, complete bridging between the bony ends is seen along the border of the radius after $S_{P I}$ implantation (Figure 8). It is due to the countless microchannels across the inner scaffold, which was very favorable for substance exchange to avoid cell necrosis in the core. Quantitatively, the mean percentages of newly formed bone filling the segmental defect region were $17.21 \%$ for the defect group without scaffold implantation, $75.38 \%$ for $\mathrm{S}_{\mathrm{MM}}$, and $92.22 \%$ for $\mathrm{S}_{\mathrm{PI}}$, which is the highest among the three groups. The Lane-Sandhu X-ray scores of the three groups (Figure 9) after 12 weeks revealed that the $S_{\text {PI }}$ group had the highest score and the blank group had the lowest one. The scores across the three groups were significantly different $(p<0.05)$. All results are due to the scaffold with microchannels that can improve cell attachment, proliferation, and differentiation.
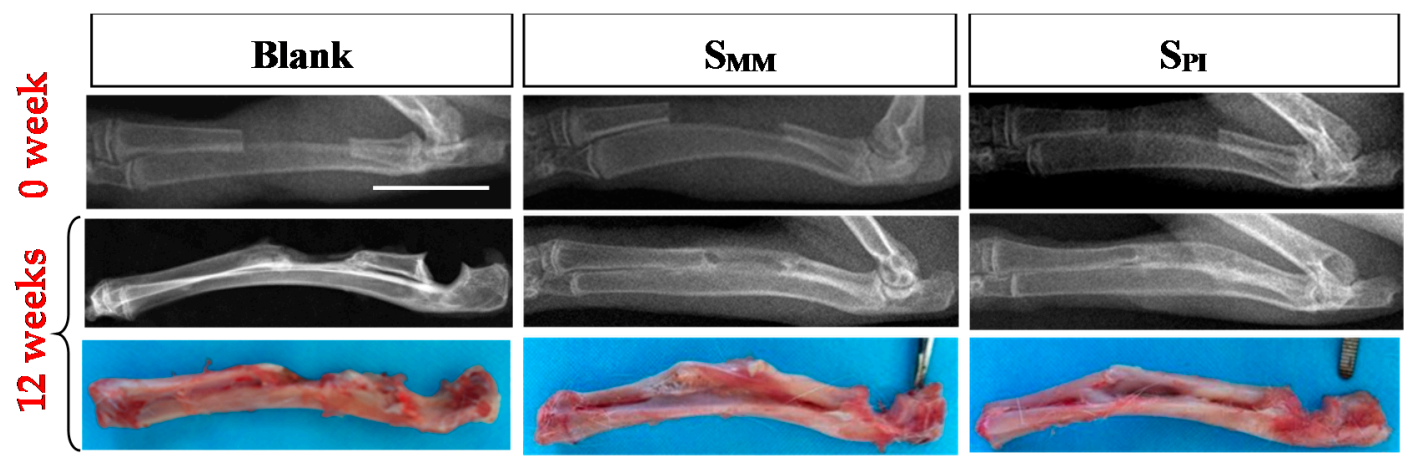

Figure 8. DR of rabbit radius defect at baseline; DR and macroscopic observation in Blank, $\mathrm{S}_{\mathrm{MM}}$, and $\mathrm{S}_{\mathrm{PI}}$ groups at 12 weeks post-surgery. The bar length $=20 \mathrm{~mm}$.

\section{Conclusions}

The use of biomaterials to repair bone defects is a long and complicated process. This process depends on porosity and the ability to allow bone ingrowth [25]. In this study, a kind of porous scaffold with microscale channels was fabricated with phase inversion. The special structure was very favorable for the movement of nutrients to and waste products from the cells in the pores, which relies on molecular diffusion. Because of this, the cells can migrate and populate in the core of the 
scaffold. In addition, the stiffness and morphology of the pore surface facilitate cell attachment and cell differentiation. Therefore, the 3D porous scaffold with microchannels was a promising option for bone defect repair.

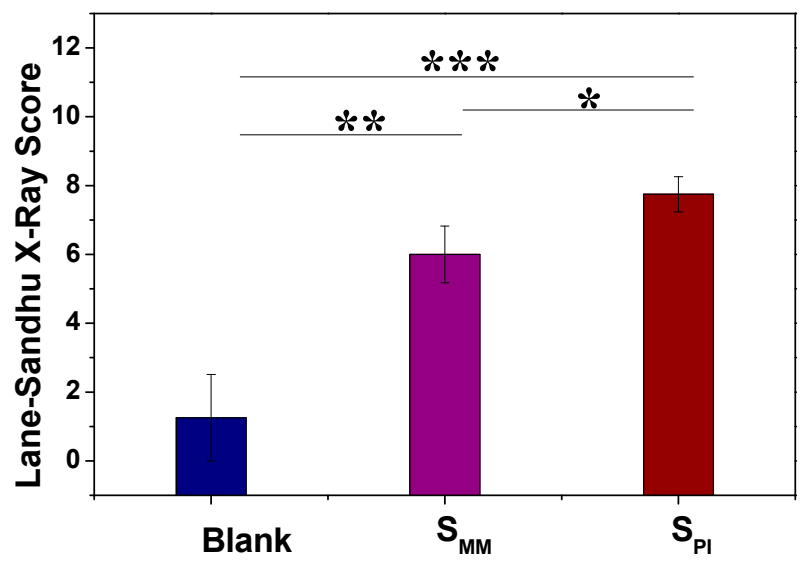

Figure 9. Lane-Sandhu X-ray scores in Blank, $\mathrm{S}_{\mathrm{MM}}$, and $\mathrm{S}_{\mathrm{PI}}$ groups at 12 weeks post-surgery. Data are presented as mean $\pm \operatorname{SD}\left(n=6,{ }^{*} p<0.05 ;{ }^{* *} p<0.01 ; * * * p<0.001\right)$.

Acknowledgments: This research was financially supported by the National Natural Science Foundation of China (No. 51307174), the Scientific Development Program of Jilin Province (Nos. 20140520050JH and 20140309005GX), the Science and Technology Planning Project of Changchun City (No. 14KG045), and the Science Foundation of the Second Hospital of Shandong University (Nos. S2015010009 and S2015010010)

Author Contributions: All authors made contributions to the development of this manuscript. Yong Hu and Jianxun Ding conceived and designed the experiments; Ning Zhang performed the experiments; Ning Zhang and Wenpeng $\mathrm{Xu}$ analyzed the data; Jianxun Ding, Ning Zhang and Yang Wang wrote and revised the manuscript.

Conflicts of Interest: The authors declare no conflict of interest.

\section{References}

1. Wu, S.; Liu, X.; Yeung, K.W.K.; Liu, C.; Yang, X. Biomimetic porous scaffolds for bone tissue engineering. Mater. Sci. Eng. R 2014, 80, 1-36. [CrossRef]

2. Seo, S.J.; Mahapatra, C.; Singh, R.K.; Knowles, J.C.; Kim, H.W. Strategies for osteochondral repair: Focus on scaffolds. J. Tissue Eng. 2014, 5. [CrossRef] [PubMed]

3. Langer, R.; Vacanti, J.P. Tissue engineering. Science 1993, 260, 920-926. [CrossRef] [PubMed]

4. Ma, P.X.; Zhang, R.; Xiao, G.; Franceschi, R. Engineering new bone tissue in vitro on highly porous poly( $\alpha$-hydroxyl acids)/hydroxyapatite composite scaffolds. J. Biomed. Mater. Res. 2001, 54, $284-293$. [CrossRef]

5. Gloria, A.; Ronca, D.; Russo, T.; D’Amora, U.; Chierchia, M.; de Santis, R.; Nicolais, L.; Ambrosio, L. Technical features and criteria in designing fiber-reinforced composite materials: From the aerospace and aeronautical field to biomedical applications. J. Appl. Biomater. Biomech. 2011, 9, 151-163. [CrossRef] [PubMed]

6. Ellis, M.J.; Chaudhuri, J.B. Poly(lactic-co-glycolic acid) hollow fibre membranes for use as a tissue engineering scaffold. Biotechnol. Bioeng. 2007, 96, 177-187. [CrossRef] [PubMed]

7. Dunn, R.L.; English, J.P.; Cowsar, D.R.; Vanderbilt, D.P. Biodegradable in-situ forming implants and methods of producing the same. US Patent No. 4938763, 3 July 1990.

8. Go, D.P.; Palmer, J.A.; Mitchell, G.M.; Gras, S.L.; O'Connor, A.J. Porous PLGA microspheres tailored for dual delivery of biomolecules via layer-by-layer assembly. J. Biomed. Mater. Res. A 2015, 103, 1849-1863. [CrossRef] [PubMed]

9. Wan, A.C.; Mao, H.Q.; Wang, S.; Leong, K.W.; Ong, L.K.; Yu, H. Fabrication of poly(phosphoester) nerve guides by immersion precipitation and the control of porosity. Biomaterials 2001, 22, 1147-1156. [CrossRef]

10. Shin, K.C.; Kim, B.S.; Kim, J.H.; Park, T.G.; Nam, J.D.; Lee, D.S. A facile preparation of highly interconnected macroporous PLGA scaffolds by liquid-liquid phase separation II. Polymers 2005, 46, 3801-3808. [CrossRef] 
11. Feng, J.H.; Park, T.G.; Lee, D.S. A facile preparation of highly interconnected macroporous poly(D,L-lactic acid-co-glycolic acid) (PLGA) scaffolds by liquid-liquid phase separation of a PLGA-dioxane-water ternary system. Polymers 2003, 44, 1911-1920.

12. Oh, S.H.; Lee, J.H. Fabrication and characterization of hydrophilized porous PLGA nerve guide conduits by a modified immersion precipitation method. J. Biomed. Mater. Res. A 2007, 80, 530-538. [CrossRef] [PubMed]

13. Zhang, P.B.; Hong, Z.K.; Yu, T.; Chen, X.S.; Jing, X.B. In vivo mineralization and osteogenesis of nanocomposite scaffold of poly(lactide-co-glycolide) and hydroxyapatite surface-grafted with poly(L-lactide). Biomaterials 2009, 30, 58-70. [CrossRef] [PubMed]

14. Lane, J.M.; Sandhu, H.S. Current approaches to experimental bone grafting. Orthop. Clin. North Am. 1987, 18, 213-225. [PubMed]

15. Parent, M.; Nouvel, C.; Koerber, M.; Sapin, A.; Maincent, P.; Boudier, A. PLGA in situ implants formed by phase inversion: Critical physicochemical parameters to modulate drug release. J. Control. Release 2013, 172, 292-304. [CrossRef] [PubMed]

16. Wu, L.; Zhang, J.; Jing, D.; Ding, J. “Wet-state” mechanical properties of three-dimensional polyester porous scaffolds. J. Biomed. Mater. Res. A 2006, 76, 264-271. [CrossRef] [PubMed]

17. Townsend-Nicholson, A.; Jayasinghe, S.N. Cell electrospinning: A unique biotechnique for encapsulating living organisms for generating active biological microthreads/scaffolds. Biomacromolecules 2006, 7, 3364-3369. [CrossRef] [PubMed]

18. Jayasinghe, S.N. Cell electrospinning: A novel tool for functionalising fibres, scaffolds and membranes with living cells and other advanced materials for regenerative biology and medicine. Analyst 2013, 138, 2215-2223. [CrossRef] [PubMed]

19. Agrawal, C.M.; Ray, R.B. Biodegradable polymeric scaffolds for musculoskeletal tissue engineering. J. Biomed. Mater. Res. 2001, 55, 141-150. [CrossRef]

20. Cui, H.; Wang, Y.; Cui, L.; Zhang, P.; Wang, X.; Wei, Y.; Chen, X. In vitro studies on regulation of osteogenic activities by electrical stimulus on biodegradable electroactive polyelectrolyte multilayers. Biomacromolecules 2014, 15, 3146-3157. [CrossRef] [PubMed]

21. Gao, T.; Zhang, N.; Wang, Z.; Wang, Y.; Liu, Y.; Ito, Y.; Zhang, P. Biodegradable microcarriers of poly(lactide-co-glycolide) and nano-hydroxyapatite decorated with IGF-1 via polydopamine coating for enhancing cell proliferation and osteogenic differentiation. Macromol. Biosci. 2015, 15, 1070-1080. [CrossRef] [PubMed]

22. Hanson, S.; D'Souza, R.N.; Hematti, P. Biomaterial-mesenchymal stem cell constructs for immunomodulation in composite tissue engineering. Tissue Eng. A 2014, 20, 2162-2168. [CrossRef] [PubMed]

23. Cheng, Z.A.; Zouani, O.F.; Glinel, K.; Jonas, A.M.; Durrieu, M.C. Bioactive chemical nanopatterns impact human mesenchymal stem cell fate. Nano Lett. 2013, 13, 3923-3929. [CrossRef] [PubMed]

24. Gumusderelioglu, M.; Aday, S. Heparin-functionalized chitosan scaffolds for bone tissue engineering. Carbohyd. Res. 2011, 346, 606-613. [CrossRef] [PubMed]

25. Domingos, M.; Intranuovo, F.; Russo, T.; De Santis, R.; Gloria, A.; Ambrosio, L.; Ciurana, J.; Bartolo, P. The first systematic analysis of $3 \mathrm{D}$ rapid prototyped poly( $\varepsilon$-caprolactone) scaffolds manufactured through BioCell printing: the effect of pore size and geometry on compressive mechanical behaviour and in vitro hMSC viability. Biofabrication 2013, 5, 045004. [CrossRef] [PubMed]

(C) 2016 by the authors; licensee MDPI, Basel, Switzerland. This article is an open access article distributed under the terms and conditions of the Creative Commons Attribution (CC-BY) license (http://creativecommons.org/licenses/by/4.0/). 